\title{
Types and Presentation of Refractive Error among Individuals Aged 0-30 Years: Hospital-Based Cross-Sectional Study, Yemen
}

\author{
Tawfik Saleh Mohammed Dhaiban $(\mathbb{D}),{ }^{1}$ Femina Purakaloth Ummer ${ }^{D},{ }^{2}$ \\ Hanan Khudadad $\mathbb{D}^{3},{ }^{3}$ and Shajitha Thekke Veettil $\mathbb{D i}^{3}$ \\ ${ }^{1}$ Department of Operations, Al Thumama Health Centre, Primary Health Care Corporation, Doha, Qatar \\ ${ }^{2}$ Department of Operations, Airport Health Centre, Primary Health Care Corporation, Doha, Qatar \\ ${ }^{3}$ Clinical Research Department, Primary Health Care Corporation, Doha, Qatar
}

Correspondence should be addressed to Shajitha Thekke Veettil; shajithajaleel@gmail.com

Received 11 January 2021; Revised 15 April 2021; Accepted 23 June 2021; Published 5 July 2021

Academic Editor: Rahman Shiri

Copyright (C) 2021 Tawfik Saleh Mohammed Dhaiban et al. This is an open access article distributed under the Creative Commons Attribution License, which permits unrestricted use, distribution, and reproduction in any medium, provided the original work is properly cited.

\begin{abstract}
Background. Refractive errors are the most common cause of visual impairment worldwide. Its proportion varied among societies and is considered as a public health challenge. Symptoms and signs associated with refractive errors are the most worrisome and common presentations in the general practice in eye clinics. Aim. The goal of this study was to determine the types and presentations of refractive error among the 0-30-year-old Yemeni population to aid early identification, diagnosis, referral, and treatment. Methodology. A cross-sectional study including 1,500 out-patients aged from 0 to 30 years attending the ophthalmology clinic in Sanaa, Yemen (between 2012 and 2015). All patients underwent visual acuity examination, autorefractometer, and anterior and posterior segment examination and were grouped according to type, that is, myopia, hyperopia, and astigmatism. Results. Hyperopia was the most common single diagnosis $(53.3 \%)$ followed by myopia (33.3\%). Astigmatism was uncommon as a single diagnosis (13.4\%) but commonly associated with hyperopia or myopia. Myopia was more common in males (42.9\%) than in females (25\%). Hyperopia was more in females $(62.5 \%)$ than in males $(42.9 \%)$. Age groups most affected by refractive errors were $13-18$ years $(27.7 \%), 19-24$ years $(24.8 \%)$, and $25-30$ years $(24.6 \%)$, respectively. Decreased vision $(53 \%)$ was a common presentation in myopia and astigmatism (41.5\%) and less in hyperopia (39.6\%). Headache was common in astigmatism (56\%), hyperopia (28.8\%), and myopia (17.8\%). Muscle imbalance, namely, exotropia (27.2\%), is mainly found in myopia and esotropia $(24.3 \%)$ in hyperopia. Conclusions. In addition to decreased vision, our patients with refractive errors mostly complain of headaches with clear variations with age and type of refractive error. Early identification and proper categorization of refractive errors by age, gender, and other demographics by general physicians in primary care can better deduce and make useful referrals to eye specialists.
\end{abstract}

\section{Introduction}

Refractive error is a problem with focusing light accurately on the retina due to the shape of the eye. The most common types of refractive error are near-sightedness (myopia), farsightedness (hyperopia), astigmatism, and presbyopia. Nearsightedness results in faraway objects being blurry, farsightedness, presbyopia results in close objects being blurry, and astigmatism causes objects to appear stretched out or blurry. Other symptoms may include double vision, headaches, and eye strain. Refractive errors are corrected with eyeglasses, contact lenses, or surgery [1]. The number of people globally with refractive errors has been estimated at one to two billion [2]. Rates vary between regions of the world with about $25 \%$ of Europeans and $80 \%$ of Asians affected [2]. Myopia is the most common disorder [3]. Rates among adults are between 15 and $49 \%$ while rates among children are between 1.2 and 42\% [4]. Hyperopia more commonly affects young children and the elderly $[5,6]$. Presbyopia affects most people over the age of 35 [1]. In 2013, 
660 million people (10 per 100) were estimated with refractive errors that have not been corrected. Of these, 9.5 million were blind due to the refractive error [7]. Refractive error is one of the most common causes of blindness along with cataracts, macular degeneration, and vitamin A deficiency [8].

Decreased vision and headache are common presentations associated with visual disability and refractive errors seen in general practice and at specialist eye clinics [9-11]. Myopia is a global public health problem affecting all age groups. A few epidemiological studies have shown variations within and between countries [12]. For instance, in studies in Asian countries, the prevalence in adults varies between $19.4 \%$ in Taiwan [13] to $48.1 \%$ in Indonesia [14]. In North America and Europe, prevalence varies from $33.1 \%$ in the USA [15] to as high as $49 \%$ among 40 -year-old adults in the United Kingdom [15]. Worldwide variations of myopia in children also show similar differences across countries and regions as shown from the results of the Refractive Error Study in Children [16] which was conducted in different countries using the same sampling strategies and procedures to measure refraction, and similar definitions of myopia to enable intercountry comparisons [16]. The study found differences between urban and rural children in Nepal with a progressive increase in prevalence from 5 to 15 years. In India, the prevalence appeared to decrease from rural to urban children whereas, among urban children in China, the prevalence of myopia varied from $5.7 \%$ in 5 -year-old to $30.1 \%$ in 10 -year-old to $78.4 \%$ in 15 -year-old people $[17,18]$.

According to the WHO regions, the estimated pool prevalence (EPP) of hyperopia was $4.6 \%$ (95\% CI: 3.9e5.2) in children. The lowest and highest EPP was seen in South-East Asia (2.2\%, 95\% CI: 1.2e3.3) and the Americas (14.3\%, 95\% CI: $13.4 e 15.2)$, respectively. The EPP of hyperopia was $30.6 \%$ (95\% CI: 26.1e35.2) in adults. Based on the results of the meta-analysis, Africa had the highest EPP of hyperopia (38.6\%, 95\% CI: 22.4e54.8) followed by the Americas (37.2\%, 95\% CI: 25.3e49) while Europe had the lowest EPP (23.1\%, 95\% CI: 6.1e40.2) [19].

The definition of astigmatism in epidemiologic studies has less variation. Considering the changes of astigmatism with age, in children above 1 year old, astigmatism was $14.9 \%$ (95\% CI: 12.7e17.1). According to WHO regions, the lowest estimated pool prevalence (EPP) was seen in SouthEast Asia (9.8\%) while the highest EPP was seen in the Americas (27.2\%) followed by the Eastern Mediterranean region $(20.4 \%)$. For adults, studies showed that $40.4 \%$ (95\% CI: $34.3 e 46.6)$ of adults had astigmatism. However, astigmatism showed a lot of variation in different $\mathrm{WHO}$ regions; the highest EPP astigmatism was seen in the Americas, and the lowest EPP was seen in Africa (11.4\% vs. $45.6 \%)$. However, it should be noted that only one study was conducted in the Americas. After the Americas, South-East Asia had the highest EPP of astigmatism (44.8\%, 95\% CI: 36.6e53.1) [19].

Given how common these eye defects are in clinical practice, research that helps to understand their natural history and especially local risk factors will be helpful for early diagnosis and properly targeted treatments in primary care practice. Therefore, the focus of this study was to determine the types and presentations of refractive errors among the $0-30$-year-old Yemeni population a basis for developing preventive interventions.

\section{Methods}

2.1. Study Design and Setting. This was a cross-sectional clinical epidemiological study involving a convenient purposive sample of patients referred from across the country for ophthalmic consultation at the department of ophthalmology, Saudi-German Hospital, a major referral center for eye problems in Sanaa, Yemen. During the study period, all patients with eye problems are referred to the Saudi-German Hospital due to the limited facilities across the health centers in Yemen.

2.2. Participants. This was a purposive sample whose selection was based on their willingness to participate in the study once they met the inclusion criteria. Given that our primary goal was to determine the types and presentation of refractive errors in our practice population, no specific sample size was calculated. No other similar studies were reported from the area on this topic.

2.3. Sample Size and Categorization. A total of 1500 patients between ages 0 and 30 years were included in this study following informed consent. Subjects were divided into the following age groups $0-6,7-12,13-18,19-25$, and 26-30 in order to include the maximum patients who agreed to participate in the study. We identified the patients who can benefit from vision rehabilitation. Then, we accurately measure visual acuity and provide appropriate vision rehabilitation services. Eligibility included referral for ophthalmic consultation by other clinicians for symptoms including decreased vision, headaches, esotropia, and exotropia. Patients, with a history of eye injury, aphakia retinoblastoma, and systemic or congenital disease were excluded from the study.

2.4. Data Sources/Measurement. Procedures: demographic data were obtained from patients' records and/or referral notes. All patients underwent visual acuity assessment and refraction measurement by cycloplegic autorefraction and subjective refraction.

Visual Acuity Testing was performed with tumbling $E$, at a distance of $6 \mathrm{~m}$. The right eye was tested first and then the left, each time with occlusion of the fellow eye. Ocular Examinations were done by a trained team consisting of an ophthalmologist. After assessment of visual acuity as above, axial length was measured by an IOL Master (version 5.02; Carl Zeiss, Jena, Germany), and a slit lamp (YZ5X; 66 Vision Tech, Suzhou, China) examination and direct ophthalmoscopy were performed by an ophthalmologist. Intraocular pressure was measured by an optometrist using noncontact tonometry (NT-1000; Nidek, Tokyo, Japan), and participants having a peripheral anterior chamber depth of 
$>1 / 2$ the thickness of cornea, IOP $25 \mathrm{~mm} \mathrm{Hg}$, given cycloplegia of one drop of $0.5 \%$ proparacaine hydrochloride in each eye followed by two drops of cyclopentolate $1.0 \%$ (Cyclogyl; Alcon, Fort Worth, TX, USA) 5 minutes apart. If the pupil size was $\ddagger 6 \mathrm{~mm}$ and the light reflex was absent after 30 minutes, cycloplegia was deemed adequate. Subsequently, autorefraction (KR-8900; Topcon, Tokyo, Japan) and subjective refraction were performed by an experienced optometrist.

A value of $6 / 6$ or $20 / 20$ is considered optimum, or perfect vision. Individuals who have $20 / 20$ vision are able to read letters that are $3 / 8$ of an inch tall from 20 feet away. Those who do not have $20 / 20$ vision are considered as refractive error. People who require spherical equivalent $(\mathrm{SE}) \leq-0.50$ Diopters of optical correction are considered as having myopia, $\mathrm{SE} \geq+2.0$. Diopters are considered having hyperopia and SE of $-2.00+3.50 \times 095$. Diopters are considered having astigmatism. Once examined, patients were grouped into the following five categories: myopia only, hyperopia only, astigmatism only, myopia with astigmatism, and hyperopia with astigmatism.

2.5. Data Collation and Analysis. Data was collated and analysed in an Excel database. Subjects were grouped according to age, gender, presenting symptoms, and diagnosis based on the five categories identified. Descriptive statistics for categorical data included frequencies (percentages). Inferential statistics were used to make comparisons between error type, frequency of occurrence, and gender difference.

2.6. Statistical Analysis. The primary goal of these analyses was to determine the types and presentation of refractive errors and their possible association with gender and age as a basis for future predictions in Yemeni children and young adults especially in general practice in primary care. All analyses were performed in Microsoft Excel and an alpha level of 0.05 was considered statistically significant. Descriptive statistics of quantitative data are presented as proportions (percentages). Categorical data, for example, age group, gender, and presenting symptoms are also presented as proportions (percentages).

\section{Results}

Out of 1500 patients, 700 were males (46.7\%) and $800 \mathrm{fe-}$ males (53.3\%). Hyperopia was the most common single diagnosis (53.3\%) followed by myopia (33.3\%). Astigmatism was uncommon as a single diagnosis (13.4\%) but commonly associated with hyperopia or myopia. Myopia was more common in males $(42.9 \%)$ than females $(25 \%)$. Hyperopia was more in females $(62.5 \%)$ than males $(42.9 \%)$ (Table 1 ). Age groups most affected by refractive errors were 13-18 years (27.7\%), 19-24 years (24.8\%), and 25-30 years (24.6\%), respectively. Decreased vision (53\%) was a common presentation in myopia and astigmatism (41.5\%) and less in hyperopia $(39.6 \%)$. Headache was common in astigmatism (56\%), hyperopia (28.8\%), and myopia (17.8\%). Muscle imbalance, namely, exotropia (27.2\%) is mainly found in myopia and esotropia (24.3\%) in hyperopia (Tables 1 and 2).

Myopia was diagnosed in 500 patients and occurred in all age groups from 0 to 30 years but was more common in males $(42.9 \%)$, compared to females $(25 \%)$. There was also a progressive increase in the frequency of myopia with age from 0-6 years (28\%) to $25-30$ years $(47.3 \%)$ (Table 1).

In contrast, hyperopia was more common among patients, 800 cases with a female preponderance $(M=42.9 \%$; $F=62.5 \%)$. In terms of age distribution, all age groups 0-30 years were affected but highest in the 13-18-year-old group (67.8\%) followed by the 19-24-year-old group (45.7\%) (Table 1).

Astigmatism was diagnosed in 200 patients with increased distribution in males (14.3\%) and females (12.5\%). No cases of astigmatism were found in the 0-6-year-old group. It was much higher in the 19-24-year-old group (18\%) compared to the other groups (Table 1).

In terms of presentation, decreased vision (53\%) was a major complaint in patients with myopia with a progressive increase in frequency from age 0-6 years from $48.6 \%$ to $57.2 \%$ in the $25-30$-year group. A similar trend was found in hyperopia but starting from a higher frequency of $33.3 \%$ in $0-6$ years with a plateau of around (47.9\%) in the age group of 25-30 years. In astigmatism, decreased vision was not found in the 0-6-year group and was less common before the age of 13 years but was a major complaint in the 25-30year group (45.5\%) and followed by 19-24-year groups (44.8\%) (Table 2).

Headache was reported less in the 7-12-year-old group (9.2\%) with myopia, increasing in the 25-30-year-old group (23.4\% highest). In hyperopia, headache was most reported in patients between 13 and 30 years amongst whom the 19-24-year group (44.1\%) complained the most followed by the 25-30-year group (40.7\%) and 13-18-year-old group (34\%). In astigmatism, there was a progressive decrease and increase in headache from 7 to 30 years old: 7-12 (57.1\%), 13-18 (58.1\%), 19-24 (55.2\%), and 25-30 (54.5\%), respectively (Table 2).

In our population, muscle imbalance, namely, exotropia, a form of strabismus where the eyes are deviated outward was found in $27.2 \%$ of myopia patients, and esotropia, a form of strabismus in which one or both eyes turn inward, was found in $2 \%$ of myopia patients. In myopia patients, exotropia found in all age groups $0-6(34.3 \%)$ and $7-12$ (46.1\%) and 13-18 (38.9\%), 19-24 (18.5\%) and 25-30 (19.4\%) respectively. A similar trend but much lesser frequency of exotropia was found in patients with hyperopia with the highest frequency $(9.3 \%)$ in the 25-30-year group. Exotropia was not a feature of astigmatism, except in a very small percentage of the 7-12-year-old group (14.3\%) (Table 2).

Similarly, esotropia was not found in astigmatism and only found in a small proportion of patients with myopia (2\%). None of those aged between 13 and 30 years with myopia presented with esotropia. However, esotropia was a common feature in hyperopia, most common in the much earlier age groups $0-6(61.1 \%)$ and 7-12-year-old group $(51.7 \%)$. It was also found in the $13-18$-year-old (21.4\%) and 
TABLe 1: Age and sex distribution of different types of refractive errors in a Yemeni population $0-30$ years $(N=1,500)$

\begin{tabular}{|c|c|c|c|c|c|c|c|}
\hline \multirow[t]{2}{*}{ Subgroup } & \multirow[t]{2}{*}{ Sample } & \multicolumn{2}{|c|}{ Myopia $(N=500)$} & \multicolumn{2}{|c|}{ Hyperopia $(N=800)$} & \multicolumn{2}{|c|}{$\begin{array}{l}\text { Astigmatism } \\
(N=200)\end{array}$} \\
\hline & & $N$ & $\%$ & $N$ & $\%$ & $N$ & $\%$ \\
\hline \multicolumn{8}{|l|}{ Age group } \\
\hline $0-6$ & 125 & 35 & 28.0 & 90 & 72.0 & 0 & 0 \\
\hline $7-12$ & 220 & 65 & 29.5 & 120 & 54.6 & 35 & 15.9 \\
\hline $13-18$ & 413 & 90 & 21.8 & 280 & 67.8 & 43 & 10.4 \\
\hline $19-24$ & 372 & 135 & 36.3 & 170 & 45.7 & 67 & 18.0 \\
\hline $25-30$ & 370 & 175 & 47.3 & 140 & 37.8 & 55 & 14.9 \\
\hline \multicolumn{8}{|l|}{ Sex } \\
\hline Male & 700 & 300 & 42.9 & 300 & 42.9 & 100 & 14.3 \\
\hline Female & 800 & 200 & 25.0 & 500 & 62.5 & 100 & 12.5 \\
\hline Total & 1500 & 500 & 33.3 & 800 & 53.3 & 200 & 13.4 \\
\hline
\end{tabular}

TABLE 2: Frequency of presenting symptoms and signs (\%) for all types of refractive error according to age group $(N=1,500)$.

\begin{tabular}{|c|c|c|c|c|c|c|c|c|c|c|}
\hline & \multirow{2}{*}{ Age group } & \multirow{2}{*}{ Sample } & \multicolumn{2}{|c|}{${ }^{*} \mathrm{DV}$} & \multicolumn{2}{|c|}{${ }^{*} \mathrm{HA}$} & \multicolumn{2}{|c|}{${ }^{*} \mathrm{Ex}$} & \multicolumn{2}{|c|}{${ }^{*}$ Es } \\
\hline & & & $N$ & $\%$ & $N$ & $\%$ & $N$ & $\%$ & $N$ & $\%$ \\
\hline \multirow{6}{*}{ Myopia } & $0-6$ & 35 & 17 & 48.6 & 0 & 0 & 12 & 34.3 & 6 & 17.1 \\
\hline & $7-12$ & 65 & 25 & 38.5 & 6 & 9.2 & 30 & 46.1 & 4 & 6.2 \\
\hline & $13-18$ & 90 & 45 & 50 & 10 & 11.1 & 35 & 38.9 & 0 & 0 \\
\hline & $19-24$ & 135 & 78 & 57.8 & 32 & 23.7 & 25 & 18.5 & 0 & 0 \\
\hline & $25-30$ & 175 & 100 & 57.2 & 41 & 23.4 & 34 & 19.4 & 0 & 0 \\
\hline & Total & 500 & 265 & 53 & 89 & 17.8 & 136 & 27.2 & 10 & 2 \\
\hline \multirow{6}{*}{ Hyperopia } & $0-6$ & 90 & 30 & 33.3 & 0 & 0 & 5 & 5.6 & 55 & 61.1 \\
\hline & $7-12$ & 120 & 50 & 41.7 & 3 & 2.5 & 5 & 4.1 & 62 & 51.7 \\
\hline & $13-18$ & 280 & 100 & 35.7 & 95 & 34 & 25 & 8.9 & 60 & 21.4 \\
\hline & $19-24$ & 170 & 70 & 41.2 & 75 & 44.1 & 10 & 5.9 & 15 & 8.8 \\
\hline & $25-30$ & 140 & 67 & 47.9 & 57 & 40.7 & 13 & 9.3 & 3 & 2.1 \\
\hline & Total & 800 & 317 & 39.6 & 230 & 28.8 & 58 & 7.3 & 195 & 24.3 \\
\hline \multirow{6}{*}{ Astigmatism } & $0-6$ & 0 & 0 & 0 & 0 & 0 & 0 & 0 & 0 & 0 \\
\hline & $7-12$ & 35 & 10 & 28.6 & 20 & 57.1 & 5 & 14.3 & 0 & 0 \\
\hline & $13-18$ & 43 & 18 & 41.9 & 25 & 58.1 & 0 & 0 & 0 & 0 \\
\hline & $19-24$ & 67 & 30 & 44.8 & 37 & 55.2 & 0 & 0 & 0 & 0 \\
\hline & $25-30$ & 55 & 25 & 45.5 & 30 & 54.5 & 0 & 0 & 0 & 0 \\
\hline & Total & 200 & 83 & 41.5 & 112 & 56 & 5 & 2.5 & 0 & 0 \\
\hline
\end{tabular}

${ }^{*} \mathrm{DV}=$ decreased vision; HA = headache; $\mathrm{Ex}=$ exotropia; Es = esotropia.

19-24-year-old group $(8.8 \%)$ but very uncommon in the 25-30-year-old group (2.1\%) (Table 2). Presbyopia was not reported in our population due to the age distribution as it affects most people over the age of 35 .

\section{Discussion}

Refractive errors are the most common ocular problem affecting all age groups and considered as a public health challenge. Recent studies and $\mathrm{WHO}$ reports indicate that refractive errors are the first cause of visual impairment and the second cause of visual loss worldwide as $43 \%$ of visual impairments are attributed to refractive errors [20]. A review study showed that uncorrected refractive errors were responsible for visual impairment in 101.2 million people and blindness in 6.8 million people in 2010 [21]. Generally, refractive errors prevalence vary among the different population due to differences in their genetic background and diverse environmental factors [22]. This study determined the types and presentation of different refractive errors among children and young adults of age 0-30 years who visited the major referral center for eye problems in Sanaa, Yemen. Myopia, hyperopia, and astigmatism were common conditions affecting the ophthalmic health of the Yemeni population. We found hyperopia was the most common single diagnosis (53.3\%) followed by myopia (33.3\%) among this population and astigmatism was uncommon as a single diagnosis (13.4\%) but commonly associated with hyperopia or myopia. Myopia was more common in males (42.9\%) and less in females (25\%); however, hyperopia was more in females $(62.5 \%)$ and less in males $(42.9 \%)$. Age groups most affected by refractive errors were $13-18$ years $(27.7 \%), 19-24$ years $(24.8 \%)$, and $25-30$ years $(24.6 \%)$, respectively.

There were not any previous publications regarding the types and presentations of refractive errors in Yemen and neighboring countries. Previous studies reported a decrease in myopia and an increase in hyperopia with increasing age $[3,23-26]$. In this study, there is an increase in the frequency 
of myopia with age from 0-6 years to 25-30 years and was more common in males compared to females. In a study conducted on the Mexican population, myopia was the most common refractive error, and the proportion seemed to increase among the younger population (10 to 29 years old), but hyperopia increased among the aging population (40 to 79 years old) [24]. In our study population, hyperopia was the most common single diagnosis (53.3\%) among age groups from 0 to 30 years and is present most with the age group of 13-18 years. This difference may be due to the age distribution of our study population. Our sample size only represents children and young adults. This needs to be considered further.

According to a meta-analysis conducted in the Middle East region, the prevalence of astigmatism was 15\% (95\% CI $10,19)$ in subjects less than or equal to 15 years and $24 \%$ $(95 \%$ CI 16, 31) in those older than 15 years of age [27]. The prevalence of astigmatism in males and females less than or equal to 15 was $9.0 \%$ (95\% CI $0.7-17.3)$ and $9.9 \%$ (95\% CI 1.5-18.3), respectively, and the prevalence in males and females over 15 years of age was $31.1 \%$ (95\% CI 18.7, 43.6) and $29.6 \%$ (95\% CI 17.2, 42.1), respectively [27]. We found astigmatism was uncommon as a single diagnosis (13.4\%) but commonly associated with hyperopia or myopia. Astigmatism is higher in males (14.2\%) compared to females $(12.5 \%)$ and is associated with hyperopia and myopia. This needs to be explored further.

A review study found a higher prevalence of hyperopia in Eastern Mediterranean children than in African, Southeast Asian, and Western Pacific children [24]. A general overview of the results of a meta-analysis done by Khoshhal et al. in the Middle East reports that the Middle Eastern countries are more prevalent to have hyperopia than other parts of the world [27]. Our study also reports the same. It is likely that the Middle East may be far behind the rest of the world, especially the East Asian countries, in terms of the epidemic of myopia. The most important hypothesis seems to be that, in addition to the racial differences of this region of the world, it should be pointed out that the economic conditions of this region in the world may have caused the region not to be myopic as elsewhere in the world. Regarding a previous report, those who live more on the basis of near work or indoor activity are more prone to myopia [27].

The association of gender with refractive errors has not been well established. Some studies have reported that the prevalence of myopia is higher in men than in women $[19,28-31]$. In other studies, however, this trend was not observed [32,33]. In this study, the distribution of myopia is higher in men (42.9\%) than in women (25\%). Symptoms like decreased vision and headache distributed as decreased vision were relatively and more uniformly high in myopia. However, in hyperopia, decreased vision, there was a progressive decrease with age, the highest being in young adults, 19-30 years old. In astigmatism, decreased vision, there was a progressive increase in presentation with age, the highest being in young adults, 19-24 years old. Headache was more common above 12 years in myopia, hyperopia, and astigmatism conditions. Fewer patients with myopia complained of headaches, with a peak age group of 25-30 years. Patients of 13 years and above with hyperopia presented with headaches and those who are 13-18 years old complained most. Children 7-12 years old with astigmatism complained most of headaches. And conditions like exotropia and esotropia were fewer common complaints in all three conditions and at different ages. However, patients with myopia were more likely to also have exotropia, especially older children 7-12 years. In hyperopia, exotropia is high in the 13-18 year-old group and those with astigmatism rarely presented with exotropia, except for children $0-12$ years old. On the contrary, esotropia was most common in children 0-12 years old with hyperopia and rare among those with astigmatism and myopia beyond 6 years and 12 years, respectively.

The limitations of the current study included that the selection of individuals was conducted using nonprobability sampling and did not also consider regional and occupational differences. For wider use and predictive generalization, a more randomized probability population sample may be necessary. Thus, despite possible limitations, a sample of patients who visited the ophthalmic clinic for any laboratory service or visual examination from different parts of the Yemen was used for the examination of refractive errors, which ensures some representation of the population. Furthermore, the types and presentations of refractive error in children and young adults have not been previously studied in Yemen. To our knowledge, this is the first report of refractive error in the Yemeni population.

\section{Conclusion}

The information of this study can be used to characterize and potentially predict types of refractive error among Yemeni children and young adults. The findings may be found useful among primary care and general practitioners and eye specialists and could help in the development of simple diagnostic tools such as clinical algorithms to aid early diagnosis and management.

\section{Data Availability}

The datasets generated and/or analysed during the current study are not publicly available but are available from the corresponding author on reasonable request.

\section{Ethical Approval}

This study was approved by the Department of Ophthalmology, Saudi-German Hospital, a major referral center for eye problems in Sanaa, Yemen.

\section{Consent}

Appropriate consent was taken from the participants for enrolment.

\section{Conflicts of Interest}

The authors declare that they have no conflicts of interest. 


\section{Authors' Contributions}

Tawfik Saleh Mohammed Dhaiban contributed to the conception and design of the study, the acquisition of data, and drafting of manuscript writing. Femina Purakaloth Ummer contributed to the manuscript writing and final approval of the manuscript version submitted for publication. Hanan Khudadad contributed to the analysis and interpretation of data and final approval of the manuscript version submitted for publication. Shajitha Thekke Veettil contributed to the interpretation of data and manuscript writing, revised it critically for content, and gave final approval to the manuscript version.

\section{Acknowledgments}

The authors wish to acknowledge Dr. Paul Amuna, Dean of the School of Public Health, University of Health and Allied Sciences, Ghana Medical School, and the Faculty of Medicine, University of Sheffield, for the statistical support, interpretation of data, and mentorship provided for the study.

\section{References}

[1] Facts About Refractive Errors [cited October 2010. Available from: https://www.nei.nih.gov/learn-about-eye-health/eyeconditions-and-diseases/refractive-errors.

[2] A. K. O. Denniston and P. I. Murray, Philip Oxford Handbook of Ophthalmology, Oxford University Press, Oxford, UK, 2018.

[3] P. J. Foster and Y. Jiang, "Epidemiology of myopia," Eye, vol. 28, no. 2, pp. 202-208, 2014.

[4] C.-W. Pan, D. Ramamurthy, and S.-M. Saw, "Worldwide prevalence and risk factors for myopia," Ophthalmic and Physiological Optics, vol. 32, no. 1, pp. 3-16, 2012.

[5] V. D. Castagno, A. G. Fassa, M. L. V. Carret, M. A. P. Vilela, and R. D. Meucci, "Hyperopia: a meta-analysis of prevalence and a review of associated factors among school-aged children," BMC Ophthalmology, vol. 14, no. 1, p. 163, 2014.

[6] T. Grosvenor, Primary Care Optometry, Elsevier, Amsterdam, Netherlands, 5th edition, 2007.

[7] Global Burden of Disease Study 2013 Collaborators, "Global, regional, and national incidence, prevalence, and years lived with disability for 301 acute and chronic diseases and injuries in 188 countries, 1990-2013: a systematic analysis for the global burden of disease study 2013," Lancet, vol. 386, no. 9995, pp. 743-800, 2015.

[8] C.-W. Pan, M. Dirani, C.-Y. Cheng, T.-Y. Wong, and S.-M. Saw, "The age-specific prevalence of myopia in Asia," Optometry and Vision Science, vol. 92, no. 3, pp. 258-266, 2015.

[9] R. Gil-Gouveia and I. P. Martins, "Headaches associated with refractive errors: myth or reality?" Headache: The Journal of Head and Face Pain, vol. 42, no. 4, pp. 256-262, 2002.

[10] M. P. Ranka and M. A. Steele, "Esotropia associated with high myopia," Current Opinion in Ophthalmology, vol. 26, no. 5, pp. 362-365, 2015.

[11] K. M. M. Breslin, L. O’Donoghue, and K. J. Saunders, “An investigation into the validity of self-reported classification of refractive error," Ophthalmic and Physiological Optics, vol. 34, no. 3, pp. 346-352, 2014.

[12] B. A. Holden, T. R. Fricke, D. A. Wilson et al., "Global prevalence of myopia and high myopia and temporal trends from 2000 through 2050," Ophthalmology, vol. 123, no. 5, pp. 1036-1042, 2016.

[13] L. L. Lin, C. J. Chen, P. T. Hung, and L. S. Ko, "Nation-wide survey of myopia among schoolchildren in Taiwan, 1986," Acta Ophthalmologica Supplement, vol. 185, pp. 29-33, 1988.

[14] M. H. Edwards and C. S. Lam, "The epidemiology of myopia in Hong Kong," Annals of the Academy of Medicine, Singapore, vol. 33, no. 1, pp. 34-38, 2004.

[15] W. S. H. Goh and C. S. Y. Lam, "Changes in refractive trends and optical components of Hong Kong Chinese aged 19-39 years," Ophthalmic and Physiological Optics, vol. 14, no. 4, pp. 378-382, 1994.

[16] R. Saxena, P. Vashist, R. Tandon et al., "Incidence and progression of myopia and associated factors in urban school children in Delhi: the North India myopia study (NIM study)," PLoS One, vol. 12, no. 12, p. e0189774, 2017.

[17] P.-C. Wu, H.-M. Huang, H.-J. Yu, P.-C. Fang, and C.-T. Chen, "Epidemiology of myopia," Asia-Pacific Journal of Ophthalmology, vol. 5, no. 6, pp. 386-393, 2016.

[18] N. Relhan, S. Jalali, N. Pehre, H. L. Rao, U. Manusani, and L. Bodduluri, "High-hyperopia database, part I: clinical characterisation including morphometric (biometric) differentiation of posterior microphthalmos from nanophthalmos," Eye, vol. 30, no. 1, pp. 120-126, 2016.

[19] H. Hashemi, A. Fotouhi, A. Yekta, R. Pakzad, H. Ostadimoghaddam, and M. Khabazkhoob, "Global and regional estimates of prevalence of refractive errors: systematic review and meta-analysis," Journal of Current Ophthalmology, vol. 30, no. 1, pp. 3-22, 2018.

[20] D. Pascolini and S. P. Mariotti, "Global estimates of visual impairment: 2010," British Journal of Ophthalmology, vol. 96, no. 5, pp. 614-618, 2012.

[21] K. S. Naidoo, J. Leasher, R. R. Bourne et al., "Global vision impairment and blindness due to uncorrected refractive error, 1990-2010," Optometry and Vision Science, vol. 93, no. 3, pp. 227-234, 2016.

[22] F. Gomez-Salazar, A. Campos-Romero, H. Gomez-Campaña et al., "Refractive errors among children, adolescents and adults attending eye clinics in Mexico," International Journal of Ophthalmology, vol. 10, no. 5, pp. 796-802, 2017.

[23] N. M. Rodriguez and A. F. Romero, "The prevalence of refractive conditions in Puerto Rican adults attending an eye clinic system," Journal of Optometry, vol. 7, no. 3, pp. 161-167, 2014.

[24] K. E. Lee, B. E. Klein, R. Klein, and T. Y. Wong, "Changes in refraction over 10 years in an adult population: the Beaver Dam eye study," Investigative Ophthalmology \& Visual Science, vol. 43, no. 8, pp. 2566-2571, 2002.

[25] F. H. Ferraz, J. E. Corrente, P. Opromolla, C. R. Padovani, and S. A. Schellini, "Refractive errors in a Brazilian population: age and sex distribution," Ophthalmic and Physiological Optics, vol. 35, no. 1, pp. 19-27, 2015.

[26] G. Haegerstrom-Portnoy, M. E. Schneck, L. A. Lott, S. E. Hewlett, and J. A. Brabyn, "Longitudinal increase in anisometropia in older adults," Optometry and Vision Science, vol. 91, no. 1, pp. 60-67, 2014.

[27] F. Khoshhal, H. Hashemi, E. Hooshmand et al., "The prevalence of refractive errors in the Middle East: a systematic review and meta-analysis," International Ophthalmology, vol. 40, no. 6, pp. 1571-1586, 2020.

[28] H.-M. Huang, D. S.-T. Chang, and P.-C. Wu, "The association between near work activities and myopia in children-a systematic review and meta-analysis," PLoS One, vol. 10, no. 10, p. e0140419, 2015. 
[29] K. Attebo, R. Q. Ivers, and P. Mitchell, "Refractive errors in an older population: the blue mountains eye study," Ophthalmology, vol. 106, no. 6, pp. 1066-1072, 1999.

[30] S. Y. Wu, B. Nemesure, and M. C. Leske, "Refractive errors in a black adult population: the Barbados eye study," Investigative Ophthalmology \& Visual Science, vol. 40, no. 10, pp. 2179-2184, 1999.

[31] S. Vitale, L. Ellwein, M. F. Cotch, F. L. Ferris 3rd., and R. Sperduto, "Prevalence of refractive error in the United States, 1999-2004," Archives of Ophthalmology, vol. 126, no. 8, pp. 1111-1119, 2008.

[32] J. Katz, J. M. Tielsch, and A. Sommer, "Prevalence and risk factors for refractive errors in an adult inner city population," Investigative Ophthalmology \& Visual Science, vol. 38, no. 2, pp. 334-340, 1997.

[33] L. Xu, J. Li, T. Cui et al., "Frequency of under-corrected refractive errors in elderly Chinese in Beijing," Graefe's Archive for Clinical and Experimental Ophthalmology, vol. 244, no. 7, pp. 871-873, 2006. 